\title{
On the Behavior of Broadcasting Protocols for MANETs Under Omission Faults Scenarios
}

\author{
Federal University of Bahia, \\ Computer Science Department, \\ Mechatronics Graduate Program \\ Salvador BA 40.170-110, BRAZIL
}

Talmai Brandão de Oliveira, Victor Franco Costa, Fabíola Greve

talmai@ufba.br, vfcosta@dcc.ufba.br, fabiola@dcc.ufba.br*

\begin{abstract}
Ensuring reliable communication between nodes is a major challenge in mobile ad-hoc networks due to wireless signal propagation that can be significantly affected by terrain, obstacles, battery exhaustion and node mobility. Existing broadcasting protocols for MANETs are able to deal with mobility, as well as congestion and collision, but only when under a fail-stop failure model. However, this model is not a good representative of the real scenarios of faults frequent in MANETs such as link failures, temporary network partitions, topology changes and momentary node failures. In this work we evaluate - through the aid of simulation experiments - how well MANET broadcasting protocols behave when under a more realistic failure model, which are characterized by omission faults. We also discuss their properties and behaviors when taking reliability into consideration. The study conducted here show that most protocols are highly impacted by node failures and are not capable of maintaining high delivery rate. Some even exhibit coverage levels that are unreasonable to expect from broadcasting protocols when placed in a real world scenario.
\end{abstract}

Keywords: Mobile Ad-Hoc Networks, Broadcasting, Fault-Tolerant Wireless Communication, Reliable Broadcasting

\section{Introduction}

A mobile ad hoc network (MANET) is a special kind of network where the mobile hosts (also called nodes) are capable of communication restricted to their wireless transmission range. Thus they are only able to communicate directly with neighboring nodes. The lack of fixed and wired gateways (base stations) forces cooperation between the nodes every time a packet has to be forwarded. Moreover, because of the shared transmission channels, nodes are not able to selectively transmit: whenever it sends a message, all of its neighbors receive it. Whenever messages overlap, collisions may occur, preventing correct reception [1].

\footnotetext{
* This work is supported by grants from CNPQ - Brazil and FAPESB - Bahia/Brazil.
} 
Broadcasting refers to the process by which one node sends messages to all other nodes in the network. It is an essential operation in all kind of networks since it may be used to collect global information, to support addressing algorithms, to implement multicasting and, particularly in MANETs, to help routing protocols to propagate routing-related information [2]. Broadcasting is an active research topic and the most significant challenge in its development is compensating between the number of messages broadcast and the number of nodes reached [3].

A large number of algorithms for broadcasting in MANETs exist. Most of them assume that during the process of broadcasting there happens none or very little topology change and that the network remains connected. But in a real scenario, this cannot always be guaranteed. This is what motivates our work. We propose to study how well existing MANET broadcasting protocols behave when under a realistic scenario. It is well known that wireless signal propagation is significantly affected by terrain, obstacles, unanticipated interference and unpredictable fading, causing constant link failures and fluctuating communication channels $[4,5]$. Many other factors also impede correct message transmission and reception as well, including hardware failure, battery exhaustion and node mobility $[6,7]$.

Previous studies on the impact of faults in broadcasting protocols have limited themselves on analyzing the impact of mobility, collisions and network congestion on the delivery rate (reliability) and on the number of gateway nodes (efficiency) [2,8]. Although these three factors can be considered as faults, they are not sufficient to denote all the possible fault scenarios that affect MANETs such as link failures, temporary network partitions, topology change during broadcasts and momentary node failures. Existing works consider failstop failures and most broadcasting protocols are tolerant to these types of failures. Furthermore, this failure model provides a simple abstraction for reasoning about failure-prone environments and system reliability, but in real systems this is not always the case. In our opinion it is the omission failure model ${ }^{1}$ that appropriately represents real fault scenarios.

We propose to study six significant broadcasting protocols. The protocols are: Simple Flooding, Dynamic Probabilistic Protocol [3], Wu and Li's protocol's [9], Scalable Broadcasting Algorithm [10], Dominant Pruning [11] and DoubleCovered Broadcast [12]. For each one of these, we discuss their properties and behaviors when taking reliability into consideration, and evaluate the impact of omission faults on their performance. Our simulation studies consist of measuring the reliability, the forwarding ratio and the end-to-end delay of the protocols when $0 \%, 5 \%, 10 \%, 20 \%, 30 \%$ and $50 \%$ of the nodes fail using an omissive failure model. As far as we know, no performance study about the impact of omission faults in broadcasting protocols has ever been done.

\footnotetext{
${ }^{1}$ In the omission model, nodes fails by crashing (prematurely halting) or by sending/receiving only a subset of the messages that it actually attempts to send/receive.
} 
Although previous simulated studies show that the broadcast protocols are very mobile resilient and support well congestion and collisions, the study conducted here show that these protocols are not fault tolerant when omission failures are taken into account. They are not capable of maintaining high delivery rate when placed in a real world scenario. The choice of the protocols leads us to believe that this conclusion can be extended to most, if not all, broadcasting protocols for MANETs which are based on the same reliability mechanisms. Based on the study conducted, we investigate the source of existing broadcasting problems and list the lessons learned as a step towards enhancing the capability of broadcasting algorithms to deal with omission faults in scalable scenarios.

This paper is organized as follows. Section 2 describes the protocols in study. Section 3 presents the simulation model, the results and lessons learned with the study. Finally, Section 4 concludes the paper and presents future works.

\section{Broadcasting in MANETs}

Since MANETs are dynamic in nature, global information exchange are no longer reasonable to expect and support. Nodes must then somehow limit themselves to local information on topology in order to broadcast. Broadcasting protocols must also be able to adapt to a wide range of MANETs including partition-less scenarios, eventually disconnected scenarios (where partitions occur rarely but reconnect quickly) and eventually connected scenarios (where partitions occur most of the time, eventually reconnect, but quickly partition once again). Broadcasting protocols are commonly classified based on their delivery guarantees, and they can either be probabilistic or deterministic. Probabilistic broadcasting protocols are those that guarantee delivery with a certain probability. Probabilistic protocols have less constraints and assumptions when compared to deterministic protocols; are usually simpler to implement; and normally have little memory requirements. Deterministic protocols on the other hand are those which assume non-probabilistic delivery guarantees. Deterministic broadcasting protocols can be further classified as either self-pruning or neighborhood designating. In selfpruning algorithms a node that receives a message decides by itself whether it is a forwarding node (also known as gateway). While in neighborhood designating algorithms it is the sending node who selects the neighboring nodes that should become gateways by piggy-backing this list in the broadcast message.

If the topology of the network is known and static, it is possible to calculate the minimum connected dominating set (namely, MCDS) [13] in order to select the set of gateways with which the smallest overhead of retransmissions can achieve the highest delivery rate. A MCDS is the smallest set of forwarding nodes such that every node in the set is connected, and all nodes which are not in the set are within transmission range of at least one node in the MCDS. Once found, the process of forwarding messages can be handled by the nodes within the set. Unfortunately, the problem of finding a MCDS has been proven to be NPcomplete [3,13], thus the use of efficient approximation algorithms is necessary. Among various alternative approximation approaches, many protocols utilizes 2- 
hop neighborhood information to reduce redundant transmission. Updated local topology information comes at a small price since by periodically sending "Hello" messages, nodes are able to construct a local view of their neighbors. But this information can be imprecise and inconsistent, since between any two "Hello" messages, a node may move, its neighbors may crash, a link may become unstable or many other situations may arise.

\subsection{Description of Chosen Protocols}

In the following paragraphs we will describe each of the chosen protocols. Among such a large number of existing broadcasting protocols for MANETs, it has been no easy task to choose the few which will be used in our study. We later justify our choices.

Simple Flooding. This is one of the simplest solutions to broadcasting. In this approach every message received by a node is forwarded. In fact, flooding (and all probabilistic protocols) are seen as an option to tackle the lack of determinism of MANETs by applying a non-deterministic solution. While there exists many papers that use this naive approach, it has been shown in $[14,15]$ that it leads to unreasonable high contention, collision and redundancy problems, which may possibly interfere in the coverage (number of individual nodes that received a specific message) and increase latency of the broadcast. This is known as the broadcast storm problem [14]. Although for a more static scenario it is not recommended, many extremely mobile and dynamic scenarios can only rely on this approach to broadcast. Actually, flooding is used by many existing broadcasting protocols as a last resort when "all else fails" [16].

Dynamic Probabilistic Approach. In order to reduce the number of forward nodes in the flooding approach, one alternative probabilistic solution proposed in [14] is that each node be allowed to re-transmit based on a probability $P$. Clearly, when $P=1$ it will behave as flooding. Most approaches to probabilistic broadcasting assume a fixed probability $[2,14]$. Depending on the value chosen a high ratio of delivery can be obtained. Another option proposed in [14] was to use a counter to keep track on the number of times a message has been received. If after a random delay the counter equals an internal counter threshold, it is assumed that the message has been received by all neighbors and the node will not retransmit. Thus, in a dense area of the network, some nodes will not rebroadcast, while in sparse areas of the network, all nodes rebroadcast. Zhang and Agrawal proposed the dynamic probabilistic approach [3] by combining the probabilistic approach with the counter based approach [14] and adjusting the value of $P$ according to the density of the network. The re-transmission probability $P$ is lowered whenever a node is positioned in a high-density area, while it is raised when in sparser areas. Network density is estimated by using an internal counter that increases whenever a node detects a neighbor and decreases periodically. 
Wu and Li. Wu and Li [9] proposed a deterministic self-pruning algorithm to calculate a set of forward nodes that form a connected dominating set. Their solution reduces the number of forwarding nodes while maintaining a high delivery ratio, and is scalable to many diverse network scenarios. Their marking process is simple and relies on constant neighborhood set exchange between nodes: a node is marked as a gateway if it has two neighbors that are not directly connected. Clearly, after neighborhood set exchange, each node knows its 2-hop neighbors. The algorithm uses a constant number of rounds to calculate the connected dominating set, which is directly related to the number of neighbors each node has. Additionally, it also uses pruning rules to reduce even further the set of gateway nodes. Their solution establishes priorities between nodes by using individual node IDs and degree (number of 1-hop neighboring nodes). The priority values are used in order to establish a total order among all nodes of the MANET. Wu and Li's protocol is well known and has been used and extended by many others $[8,17,18]$. But these works where all inspired on reducing the number of gateways nodes and on increasing broadcasting efficiency; not necessarily on achieving high message delivery ratio. Simulation results clearly show that although older, the original protocol still ensures higher message coverage [8]. This is why we chose it over the newer protocols.

Scalable Broadcast Algorithm (SBA). The main idea of the deterministic self-pruning scalable broadcasting algorithm proposed by Peng and Lu (namely, SBA) [10] is that a node does not need to rebroadcast a message that already has been received by neighboring nodes. In order to determine this, each node needs to have knowledge of local 2-hop topology and of duplicate messages. Their algorithm works in 2 steps: local neighborhood discovery and data broadcasting. Local neighborhood discovery consists of exchanging neighborhood sets between local nodes in order to learn 2-hop topology information (exactly like Wu and Li's protocol). For data broadcasting, whenever a node $t$ receives a message $m$ from his neighbor $v$, before forwarding the message it checks which nodes belong to $v$ 's neighborhood. Since $v$ transmitted, node $t$ knows all the nodes that should have received the message. By looking at its own neighborhood set, $t$ can determine if there are still any other neighbors which have not received $m$. Only when there exists neighbors in this situation will $t$ schedule a re-transmission. Instead of immediately re-transmitting, the authors proposed a random backoff delay based on the density of the neighborhood. Nodes with more neighbors will have a higher priority and will rebroadcast earlier, thus raising the chances of a single transmission reaching a greater number of nodes.

Dominant Pruning (DP). The dominant pruning algorithm (namely, DP) [11] is a deterministic neighborhood designating broadcasting protocol that uses 2-hop neighborhood information to reduce redundant transmissions. In DP, whenever a node receives a message, it selects the smallest number of forwarding nodes that can cover all nodes in a 2-hop distance. That is, when node $j$ receives a message from node $k$, it selects from the set $N(j)$ the minimum number of 
nodes that should act as gateways to reach all nodes in $N(N(j))$. The DP protocol assumes that when node $k$ first transmitted the message, all of its 1-hop neighbors (which is the set $N(k)$ ) correctly received the message. It also assumes that when node $j$ forwards the message, all of its 1-hop neighbors $(N(j))$ will correctly receive the message as well. Thus, node $j$ will then just try to determine $N(N(j))-N(j)-N(k)$ (which supposedly will be the remaining nodes who, after node $j$ forwards, will not have received the message yet). By determining this, it will then loop through $N(j)$ and select the smallest number of nodes that are able to guarantee coverage. These nodes will become forwarding nodes. Since it is a neighborhood designating protocol, it piggybacks this list in the broadcast message. Although there exist newer algorithms that extend DP, such as [19] where simulation results show that neighborhood information is more effectively used (lower number of gateway nodes) and even more redundant messages are eliminated, they unfortunately seem to produce results which have lower delivery rates. This obviously makes sense since it is the redundant messages that help raise message coverage. Once again we prefer the original protocol with higher coverage for this work.

Double-Covered Broadcast (DCB). Lou and Wu's goal when proposing the double-covered broadcasting protocol (namely, DCB) [12] was to reduce the number of forwarding nodes (increase efficiency) without sacrificing the broadcast delivery ratio (reliability). It is classified as a neighborhood designating protocol, much like dominant pruning. By selecting a set of gateway nodes where not only every 2-hop node is covered, but also where all 1-hop nodes are covered by at least 2 forwarding neighbors (the sender itself and one of the selected gateway nodes), it benefits from the broadcast redundancy to improve reliability. Additionally, in DCB the re-transmission of the message by the gateways node serves as an ack of correct message reception to the original sending node. This scheme avoids the ACK implosion problem [20]. The sender will wait during a pre-determined time to overhear the re-transmissions by every chosen gateway node. If it fails to detect all of the re-transmissions it assumes that a transmission failure occurred. The sender will keep re-sending the message until all forward nodes have re-transmitted or until a threshold is reached. By double-covering, DCB assumes that at least two transmissions will reach the nodes, therefore this redundancy prevents a single transmission error from interfering on message transmission and reception.

Justifications. Simple flooding and Dynamic Probabilistic protocol are, in our opinion, good representations of the probabilistic approach and were chosen for their high redundancy. Simple flooding seemed a natural choice for its simplicity while Dynamic Probabilistic for its novel approach for dynamically setting the re-broadcast probability. As representatives of self-pruning protocols we chose $\mathrm{Wu}$ and Li's protocol and Scalable Broadcasting Algorithm for their efficient use of neighborhood information and for their good simulation results (regarding message delivery rate). Dominant Pruning was chosen for similar reasons, but 
rather as the representative of neighborhood designating protocols. The DoubleCovered Broadcast protocol can be considered a reliable broadcasting since it tries to ensure message delivery beyond best-effort guarantees. It was chosen not only for its' novelty, but also for applying multiple reliability mechanisms.

\section{Performance Evaluation}

In order to evaluate the performance and behavior of the broadcasting protocols when in an omission-fault injected environment, we ran simulations using the NS-2 network simulator [21].

\subsection{Simulation Model}

The Scenario. Motivated by findings that simplistic mobility and radio propagation models had a significant impact on the behavior of MANET broadcasting protocols [5] and, in order to attest that our simulation results were valid, we chose more realistic parameters with which to simulate. The simulation parameters are listed in Table 1 . During the simulation the nodes were confined within $1300 \times 1300 \mathrm{~m}^{2}$. Each of them had a constant transmission range of $250 \mathrm{~m}$. For the radio propagation model we used the Two-Ray Ground Reflection model as implemented in the simulator, while the MAC layer followed the IEEE 802.11 specification with no RTS/CTS/ACK for all message transmissions. We used BonnMotion v1.3a [22] as our mobility scenario generator. The movement pattern of each node follows the Gauss-Markov mobility model as defined by BonnMotion. Up to an 18 second pause time can occur before a node moves to a new location.

\begin{tabular}{|l|r|}
\hline \multicolumn{2}{|c|}{ Simulation Parameters } \\
\hline Simulator & NS-2 $(2.30)$ \\
\hline Network Area & $1300 \times 1300 \mathrm{~m}^{2}$ \\
\hline Transmission Range & $250 \mathrm{~m}$ \\
\hline Simulation Time & $500 \mathrm{~s}$ \\
\hline \# of Trials & 20 \\
\hline Mobility Model & Gauss-Markov \\
\hline Broadcast Rate & $10 \mathrm{msg} / \mathrm{s}$ \\
\hline Node Speed & $1 \mathrm{~m} / \mathrm{s}$ \\
\hline Confidence Interval & $95 \%$ \\
\hline \multicolumn{2}{|c|}{ Table 1. Simulation Parameters }
\end{tabular}

To allow for proper initialization and settling, we allow 3000 seconds of node movement without any kind of message exchange. Each simulation then ran for a total of 500 seconds. In the first 100 seconds only "Hello" type messages are sent 
to allow for updated local topology information to be exchanged throughout the network. It is during the next 100 seconds that we configure nodes to broadcast data messages as well. For the last 300 seconds no new data messages are broadcasted, but nodes still exchange "Hello" messages, retransmit buffered messages as needed and move. This is to allow for proper message delivery termination, such as unsent queued messages, as well as possible re-transmission attempts. Each simulation was repeated 20 times to achieve at least a $95 \%$ confidence interval for the results.

Values used for the simulation such as broadcast rate and node speed were determined based on results obtained through a previous work [23]. Although well known that mobility is a major cause of delivery failure $[19,24,25]$, in the cited work node speeds where varied between $1 \mathrm{~m} / \mathrm{s}$ and $160 \mathrm{~m} / \mathrm{s}$. The choice of $1 \mathrm{~m} / \mathrm{s}$ reduces the negative effects of mobility on the protocols. In the same manner, the broadcast rate value of 10 packets $/ s$ was determined after simulation runs varying between 1 packet/s and 111 packets $/ \mathrm{s}$. This value was chosen since, on average, even when taking node failure in consideration, had the best overall effect on every metric measured and permitted the most stable and reliable communication. We refer the reader to [23] for more details.

The Metrics. We have defined three metrics with which we have divided the simulation studies. The metrics are reliability, forwarding ratio and end-to-end delay. Since our main priority is analyzing the reliability of the protocols, both energy concerns and protocol overhead-related metrics (such as "hello" message exchange) were not taken into consideration. All of the values listed below are available through logs generated by the simulator.

- Reliability. A high delivery ratio is the primary goal of any broadcast protocol, thus reliability is the most significant metric. It will demonstrate not only if the broadcast protocol in question does what it is supposed to do, but will help to show how each protocol deals with failure. Since the number of nodes participating in the simulation is known by the simulator, we are able to extract and analyze the percentage of nodes that received any given message.

- Forwarding Ratio. Protocol efficiency is given by the number of gateway nodes that re-transmit and take an active role in the broadcast. Therefore, an efficient broadcast protocol is one that uses the lowest number of gateways to reach the highest number of nodes, which in turn will lead to a lower number of packets and consequently to less congestion and collision. But with the induced failure of nodes, efficiency is better measured as a ratio of the number of nodes that received a packet to the number of nodes that acted as gateways. We denominate this the forwarding ratio.

- End-to-End Delay. Finally, end-to-end delay is a metric normally used in conjunction with the others to help understand how congestion has affected the protocols, since it measures how long it takes any given packet to reach every node. 
Fault Model. Most deterministic broadcasting protocols are resilient to failstop failures due to the fact that these protocols use constant neighborhood set exchange between nodes. Thus, a faulty node can only interfere for a short time during the broadcasting process. Shortly after the failure, all neighboring nodes will detect the fault and in future broadcasts, the node (which has now crashed), will no longer be involved in any broadcast. Using a fail-stop failure model is, in our opinion, inadequate to analyze faults when simulating deterministic broadcasting protocols. Thus, unlike any other work we have seen before, we have implemented an omission fault model in order to simulate a real world scenario characterized by interference introduced by the environment, link instability and transmission failure due to node movement. This model is also applicable to probabilistic broadcasting protocols.

In our implementation, during each one of the runs, an uniformly random selected set of nodes will fail to send and receive any kind of messages for 10 seconds. When this period is over, a new group of randomly selected nodes will be chosen to fail. The exact number of nodes chosen depends on the percentage of failed nodes which can be $0 \%$ (failure free), $5 \%, 10 \%, 20 \%, 30 \%$ and $50 \%$. When defining this fault model, we had in mind the importance of randomly choosing faulty nodes, instead of selecting a static groups of nodes. But, since the number of failures is fixed, to help better spread the failure and to represent omission faults such as the ones listed above, we periodically pick another group of nodes throughout the network.

This implements an omission fault model and also helps to stress those protocols that assume a correct behavior on the reception and transmission during a broadcast, specially by some special set of nodes such as the gateway nodes. On the other hand, this fault model will favor those protocols that use additional mechanisms to properly identify message reception by neighboring nodes. It is important to note that, in parallel with the omission faults simulated by our model, other failures still keep occurring during the execution. For example, transmitted messages frequently are dropped since, after all, the radio propagation model used (in NS-2) allows for transmission errors. Nodes are also mobile, so they may even move out of transmission range.

In order to correctly compare the protocols, all were simulated under the same set of mobility and fault patterns, including the exact same broadcast message sending times.

\subsection{Simulation Results}

We present now the simulation results for each of the defined metric. We individually compared each of the protocols with themselves in order to be able to identify how each of them behaved when under different failure scenarios, ranging from $0 \%$ to $50 \%$. Then we compared the protocols with each other under the same scenarios of faults. Note that all specific values used in the next paragraphs are mean values, but the results in the graphs have confidence intervals of $95 \%$ plotted as well, although these are extremely small and when plotted on a full scale graph, can hardly be seen. 
(a) Simple Flooding

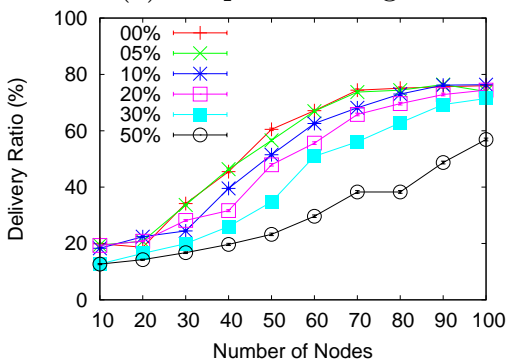

(c) $\mathrm{Wu}$ and $\mathrm{Li}$

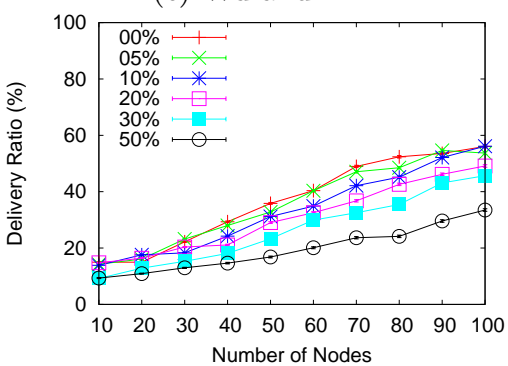

(e) Dominant Pruning (DP)

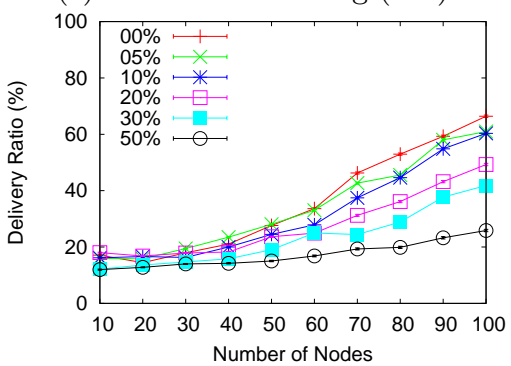

(b) Dynamic Probabilistic Broadcasting

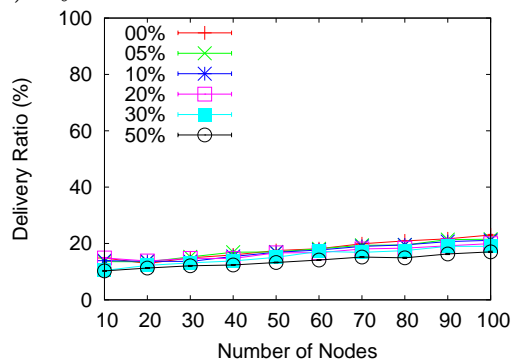

(d) Scalable Broadcasting Algorithm (SBA)

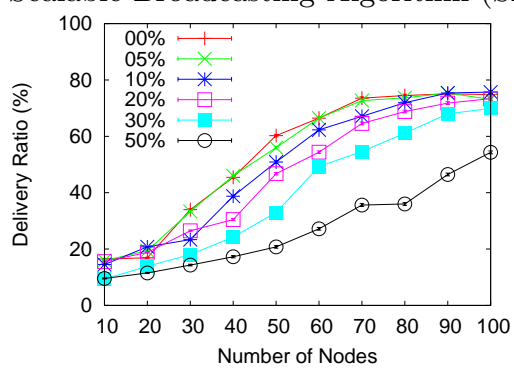

(f) Double-Covered Broadcasting (DCB)

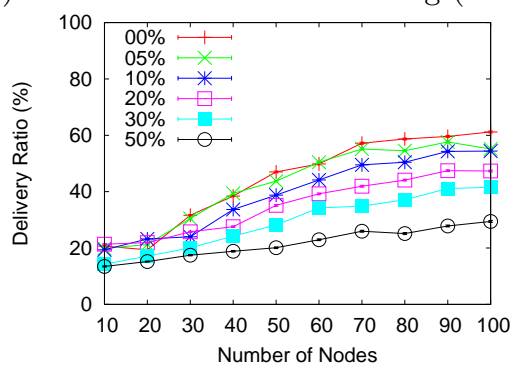

Fig. 1. The Effects of Node Failure on Reliability (Individual View)

Reliability Results. Figure 1 clearly shows what was expected: the reliability of all broadcasting protocols simulated lowers as the number of node failure increases. This conclusion is true to both deterministic and probabilistic approaches. In no scenario was any protocol able to deliver messages to more than $80 \%$ of the network. Flooding and SBA where the ones with the highest delivery ratios, reaching almost $80 \%$ of the network on a fail-free run ( $0 \%$ of node failure) in a dense network scenario. Even when failure rates raised to $30 \%$ both protocols remained pretty stable, decreasing an average of $5 \%$ in delivery rates. On the other hand, in the worst-case scenario, where $50 \%$ of the nodes failed, even in a dense network the delivery ratio barely reached $57 \%$. In Figure 2 we can realize how similar both protocols behave. In simple flooding, both reliability and faulttolerance is assumed because of the high redundancy [26]. Unfortunately, this 
does not guarantee message delivery to all nodes and only relies on the inherent redundancy to obtain coverage. SBA's drawback is that it requires up-to-date neighborhood information. Without it, unfortunately, a node that is receiving a message will erroneously calculate its forward status. Furthermore, a node has absolutely no guarantees that the same message correctly arrived at its neighbors, and therefore cannot just assume correct reception.

(a) $0 \%$ scenario

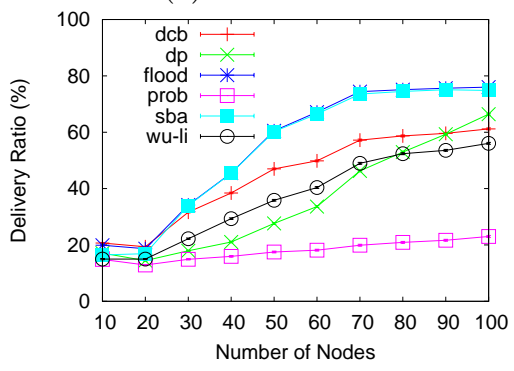

(c) $30 \%$ scenario

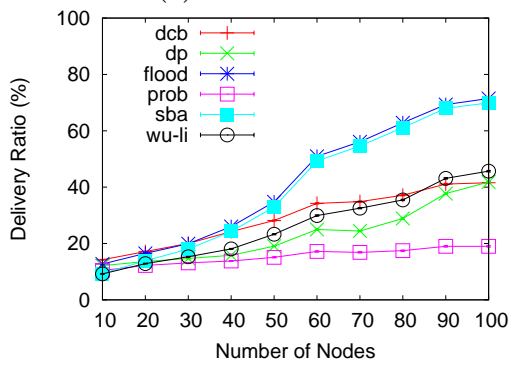

(b) $20 \%$ scenario

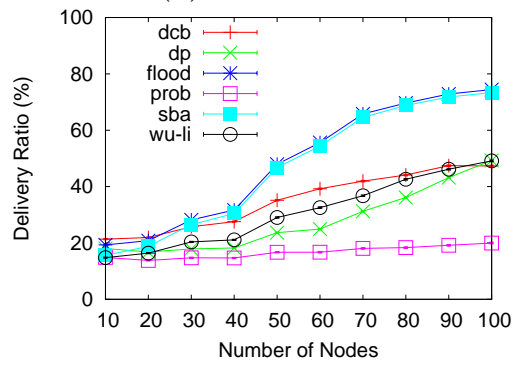

(d) $50 \%$ scenario

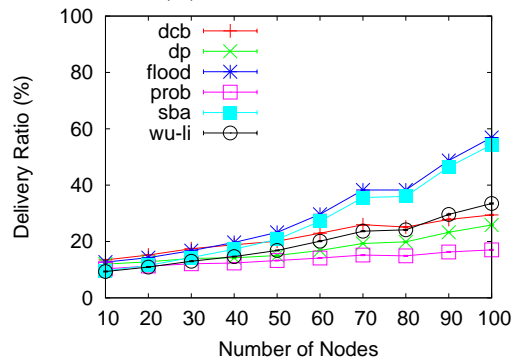

Fig. 2. The Effects of Node Failure on Reliability (Comparison View)

Dominant Pruning, Double-Covered and Wu and Li's protocols all had similar results regarding message delivery when in a dense fail-free network (with 100 nodes). DP reached $66 \%$ of the nodes, DCB $61 \%$ and $\mathrm{Wu} 56 \%$. But, when failures were introduced to the simulation, both DP and DCB were rapidly impacted by node failures, delivering messages to less than $50 \%$ of the network with as little as $20 \%$ node failure, and barely reaching $41 \%$ when $30 \%$ of the nodes failed. In the worst case ( $50 \%$ failure) neither were able to reach more than $29 \%$ of the network. Wu and Li's, on the other hand, was capable of delivering messages to $56 \%$ of the network as long as node failures remained below $10 \%$. This value decreases to about $45 \%$ when node failures increase to $30 \%$. The delivery ratio only drops to $33 \%$ when in a worst-case scenario. We can appreciate direct comparisons through the graphs of Figure 2.

The authors of DP inherently assume that no errors occur during message transmission, by accepting that when a node transmits a message, all of its 1- 
hop neighbors correctly received the message and that, when a neighboring node forwards the message, all of its 1-hop neighbors correctly receive the message as well. But, in a fault-enabled environment this is, most often, not the case and simulations result corroborate with this as perceptible consequences to message delivery can be seen. Double-Covered's approach to broadcasting, unfortunately, relies on the reception of the acknowledgment by the sender node, but this does not ensure that the 2-hop neighbors received the message as well. Both the exposed terminal problem - where an outgoing transmission collides with an incoming transmission - and the hidden terminal problem - where two incoming transmissions collide with one another - can defeat the reliability mechanism inherent in DCB.

According to $\mathrm{Wu}$ and $\mathrm{Li}$, in their protocol the resultant dominating set includes nodes of the shortest path. But, in an ad hoc environment, where the nodes are free to move, the shortest path tends to be the most unstable and prone to link failure [27]. This is not taken into consideration and no guarantees are ever made that a gateway is forwarding the messages nor is the delivery of any message ensured.

Dynamic Probabilistic delivery ratios' had the lowest values of all protocols, and were all between $25 \%$ and $17 \%$. The highest value when in a fail-free scenario, and the lowest in the worst-case. It is assumed by the protocol that the network topology does not change drastically, so that the probability calculated can be a reasonable approximation of the optional probability for the next packet transmission. This, unfortunately, is only the case for networks where movement speed is low. Furthermore, while the probability of broadcasting is dynamically adjusted, it becomes dependent upon other fixed parameters that need also be carefully selected (like for example, the exact value of timeouts).

Forwarding Ratio Results. As already stated, forwarding ratio is defined as a ratio of the number of nodes that received a packet (\#receptions) to the number of nodes that acted as gateways (\#gateways). That is, \#gateways/\#receptions. The higher the forwarding ratio, the greater the number of nodes that had to forward the message. A low forwarding ratio then means that the protocol is efficient, since it uses the lowest number of gateways to reach the highest number of nodes. However, this does not mean that an efficient protocol is also reliable. In Figure 3 we can note how the forwarding ratio of all protocols is lowered as failures are introduced to the scenarios. That is, as more nodes fail, the smaller the number of nodes involved in the forwarding process. Flooding is the most inefficient protocol, as it needs to involve almost all receiving nodes in the forwarding process. Note how in the fail-free scenario it involves 100\% of the nodes that receive a message in the forwarding process. Double-Covered Broadcasting also involves a large number of nodes in the forwarding process, and while it is more efficient than flooding, it is not much. Dynamic probabilistic obtains higher efficiency, since it compromises at most $50 \%$ of the nodes, but this drops to as low as $25 \%$ when in a $50 \%$ failure scenario. In an increasing scale, dominant pruning is the next most efficient protocol, as it also maintains node 
(a) Simple Flooding

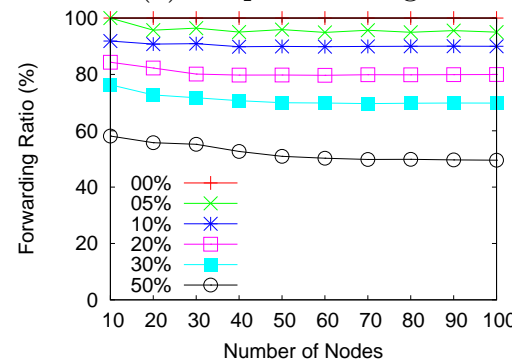

(c) $\mathrm{Wu}$ and $\mathrm{Li}$

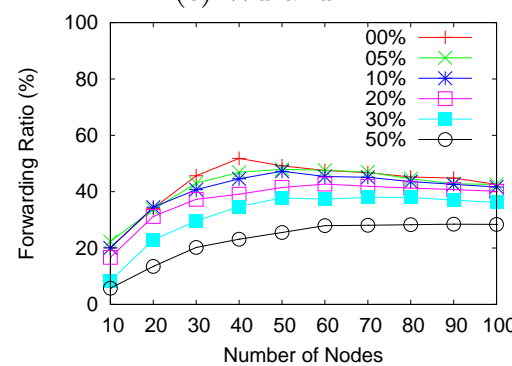

(e) Dominant Pruning (DP)

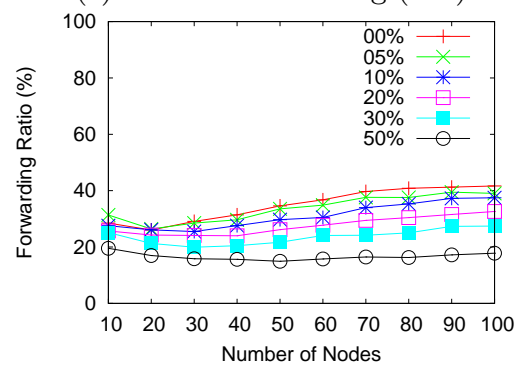

(b) Dynamic Probabilistic Broadcasting

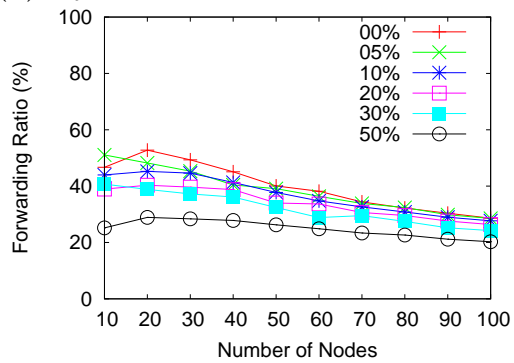

(d) Scalable Broadcasting Algorithm (SBA)

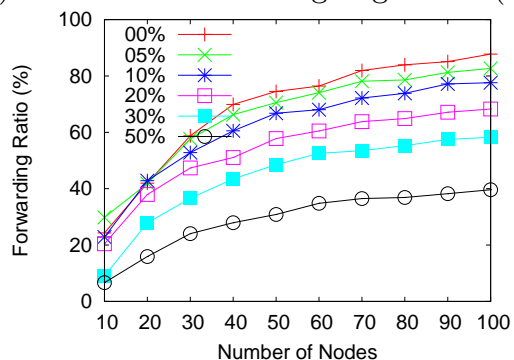

(f) Double-Covered Broadcasting (DCB)

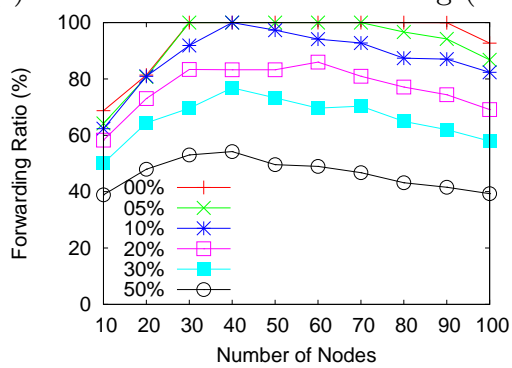

Fig. 3. The Effects of Node Failure on Forwarding Ratio (Individual View)

participation in the $25 \%-40 \%$ range, but unlike dynamic probabilistic, this happens most of the time. In the worst-case scenario ( $50 \%$ node failure) this lowers to $18 \%$. Both $\mathrm{Wu}$ and Li's protocol, as well as SBA, have high efficiency values when in a sparse and fault-enabled scenario, involving between $16 \%-25 \%$ of the network on average, but reaching values as low as $10 \%$. But similarities stop there. SBA's efficiency then drops sharply, involving between $60 \%-80 \%$ of the network. While $\mathrm{Wu}$ and Li's behavior settle between 25\% - 40\%. Through Figure 4 we are able to directly compare the protocols.

End-to-End Delay Results. All protocols, as can be seen in Figure 5, have a slight drop in the end-to-end delay as more nodes failed. This was the expected behavior since the node re-transmission activity ceased on all faulty nodes. 
(a) $0 \%$ scenario

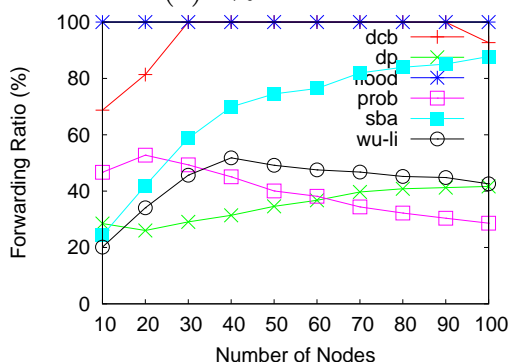

(c) $30 \%$ scenario

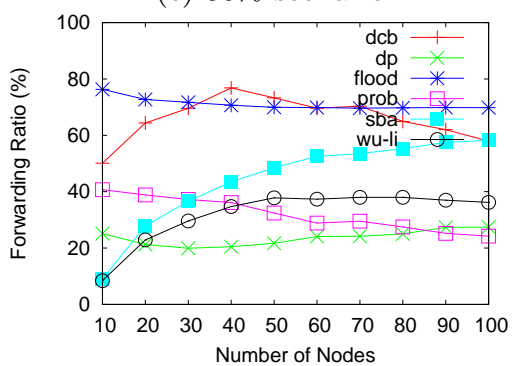

(b) $20 \%$ scenario

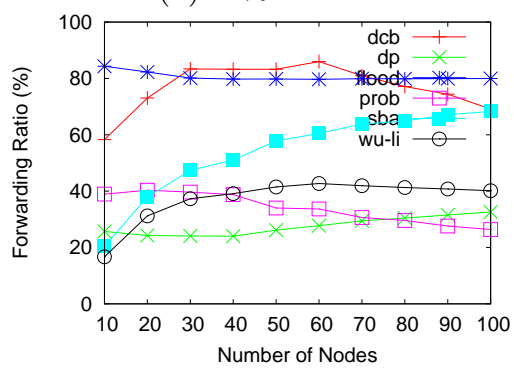

(d) $50 \%$ scenario

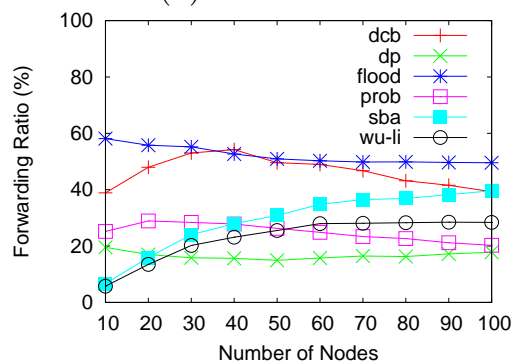

Fig. 4. The Effects of Node Failure on Forwarding Ratio (Comparison View)

Overall, DCB had the highest end-to-end delay, mostly due to the re-transmission attempts when no acknowledgement is overheard. SBA's backoff delay (to reduce congestion and collisions) produced a longer overall delay to transmit messages. Flooding, on the other hand, causes the broadcast storm problem which also increases latency of the broadcast. The remaining protocols all had low delays, with both $\mathrm{Wu}$ and $\mathrm{Li}$ and $\mathrm{DP}$ needing some time to update 2-hop neighborhood information, while dynamic probabilistic's simpler approach to broadcasting maintains latency to a minimum.

\subsection{Lessons Learned}

The biggest challenge behind broadcasting in MANETs still lies in finding the balance between message overhead (i.e. redundancy) and reliability. On one hand, a large number of re-transmissions will result in a larger number of nodes reached, but so will the chances of collisions and possibly transmission delays rise as well. On the other hand, when too small of a number of re-transmissions is chosen there is a potential risk of not all nodes being reached. The results here presented allows us to list a few lessons learned:

1. While probabilistic protocols are seen as a way to handle the lack of determinism of MANETs, improperly adjusting this class of protocols in order to inhibit redundant retransmissions can cause more loss than gains. While flooding 
(a) $0 \%$ scenario

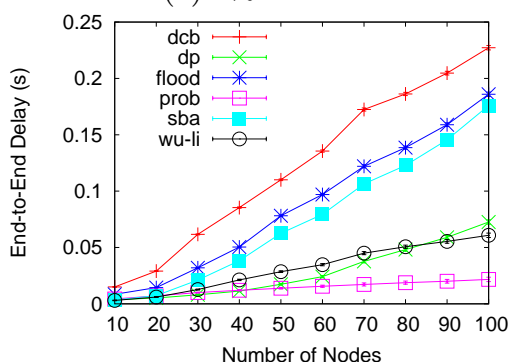

(c) $30 \%$ scenario

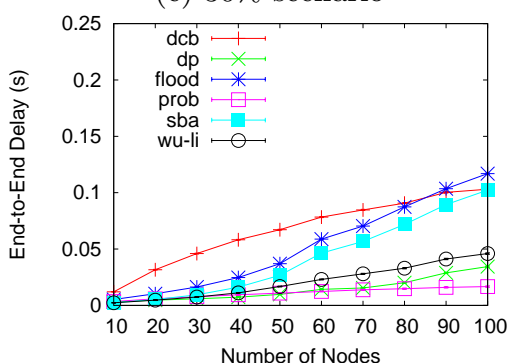

(b) $20 \%$ scenario

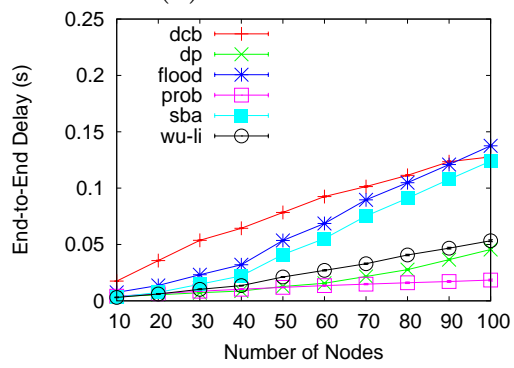

(d) $50 \%$ scenario

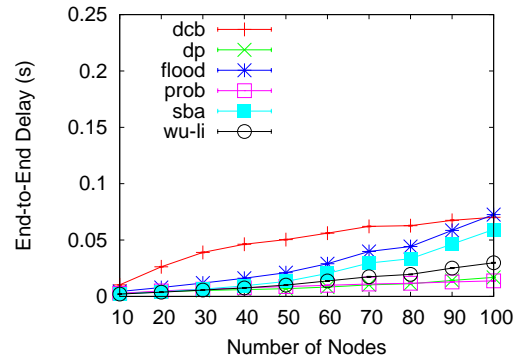

Fig. 5. The Effects of Node Failure on End-to-End Delay (Comparison View)

had high delivery ratios, dynamic probabilistic broadcasts hardly reached the intended nodes.

2. Most deterministic algorithms rely on correctly updated neighborhood knowledge in order to calculate forward status. But in a fail-prone scenario this information may be misleading. This seems to affect much more neighborhood designating protocols than self-prunning ones.

3. Additional mechanisms to properly identify message reception is recommended to determine if all 1-hop and 2-hop neighbors received a message. Nodes must not just assume correct reception. While simply overhearing a retransmission by a neighboring node is one possible solution, its use should be limited to selfpruning algorithms where more nodes can possibly detect incorrect forwardingrelated decisions.

4. Most protocols handled failures up to $10 \%$ of the network without a large impact on delivery rates. This information should be used by algorithms, especially when adjusting dynamic thresholds.

5. For reliability to be ensured, redundancy is a must. Simulations result indicate that when $60 \%$ of the network received a message, at least $25 \%$ of the network acted as gateways. And to reach $80 \%$ of the network, at least $60 \%$ of the nodes forwarded the message. Efficiency, albeit important, must not be the primary focus of a broadcasting protocol that intends to reach all correct nodes of the network. 


\section{Conclusion}

In order to evaluate the impact of faults on the performance of significant broadcasting protocols, we have conducted simulations under various network scenarios and situations. The simulation studies consisted of measuring the reliability, the forwarding ratio and the end-to-end delay of the protocols when in an omission fault injected environment. It is interesting to note that the protocols are unable to cope well with failures under the realistic model proposed. Regarding message delivery ratio, all protocols suffer a somewhat performance degradation, but a few reach levels of coverage that is unacceptable for broadcasting protocols. Based on the study conducted, we investigated the source of existing broadcasting problems and list the lessons learned as a step towards enhancing the capability of broadcasting algorithms to deal with omission faults in scalable scenarios.

Our future work includes researching possible extensions to broadcasting algorithms in order to provide efficient mechanisms to deal with faults. We also plan on extending our fault model to reflect an even more realistic environment. The current definition of our fault model takes into consideration the importance of randomly choosing faulty nodes throughout the network and represent failures characterized by interference introduced by the environment, link instability and transmission failure due to node movement. Nevertheless, it still represents a rather peculiar failure model where a certain number of nodes fail (and then start working correctly) at exactly the same time. Despite this fact, the results presented in this work are valid since they still model a scenario much more realistic than the simpler fail-stop model.

\section{Acknowledgement}

The authors would like to thank all the anonymous referees whose insightful comments helped us to improve the paper presentation.

\section{References}

1. Ray, S., Carruthers, J., Starobinski, D.: Evaluation of the masked node problem in ad hoc wireless lans. IEEE Trans. on Mobile Computing 4(5) (2005) 430-442

2. Williams, B., Camp, T.: Comparison of broadcasting techniques for mobile ad hoc networks. In: Proc. of the 3rd ACM Int. Symp. on Mob. Ad Hoc Networking \& Computing, ACM Press (2002) 194-205

3. Zhang, Q., Agrawal, D.P.: Dynamic probabilistic broadcasting in manets. Journal of Parallel and Distributed Computing 65(2) (2005) 220-233

4. Basile, C., Killijian, M., Powell, D.: A survey of dependability issues in mobile wireless networks. Technical report, LAAS CNRS Toulouse, France (2003)

5. Kotz, D., Newport, C., Gray, R.S., Liu, J., Yuan, Y., Elliott, C.: Experimental evaluation of wireless simulation assumptions. In: Proc. of the 7th ACM Int. Symp. on Modeling, Snalysis and Simulation of Wireless and Mobile Systems (MSWiM '04), New York, NY, USA, ACM Press (2004) 78-82 
6. Huang, Q., Julien, C., Roman, G.: Relying on safe distance to achieve strong partionable group membership in ad hoc networks. IEEE Transactions on Mobile Computing 3(2) (2004) 192-205

7. Stankovic, J.A., Abdelzaher, T., Lu, C., Sha, L., Hou, J.: Real-time communication and coordination in embedded sensor networks. In: Proceedings of the IEEE, 91(7). (2003)

8. Dai, F., Wu, J.: Performance analysis of broadcast protocols in ad hoc networks based on self-prunning. IEEE Transactions on Parallel and Distributed Systems 15(11) (2004) 1027-1040

9. $\mathrm{Wu}, \mathrm{J} ., \mathrm{Li}, \mathrm{H} .:$ On calculating connected dominating set for efficient routing in ad hoc wireless networks. In: DIALM '99: Proc. of the 3rd Int. Workshop on Discrete algorithms and Methods for Mobile Computing and Comm., New York, NY, USA, ACM Press (1999) 7-14

10. Peng, W., Lu, X.C.: On the reduction of broadcast redundancy in mobile ad hoc networks. In: Proc. 1st ACM international symp. on Mobile ad hoc networking \& computing (Mobihoc), Piscataway, NJ, USA, IEEE Press (2000) 129-130

11. Lim, H., Kim, C.: Flooding in wireless ad hoc networks. Computer Comm. 24(3-4) (2001) 353-363

12. Lou, W., Wu, J.: Toward broadcast reliability in mobile ad hoc networks with double coverage. IEEE Trans. on Mobile Computing 6(2) (2007) 148-163

13. Lim, H., Kim, C.: Multicast tree construction and flooding in wireless ad hoc networks. In: Proc. of the 3rd ACM Int. Workshop on Modeling, Analysis and Simul. of Wireless And Mob. Sys. (MSWIM '00), ACM Press (2000) 61-68

14. Ni, S.Y., Tseng, Y.C., Chen, Y.S., Sheu, J.P.: The broadcast storm problem in a mobile ad hoc network. In: Proc. 5th ACM/IEEE Int. Conf. on Mobile Computing and Networking, ACM Press (1999) 151-162

15. Tseng, Y.C., Ni, S.Y., Shih, E.Y.: Adaptive approaches to relieving broadcast storms in a wireless multihop mobile ad hoc network. IEEE Transactions on Computers 52(5) (2003) 545-557

16. Obraczka, K., Viswanath, K., Tsudik, G.: Flooding for reliable multicast in multihop ad hoc networks. Wireless Networks 7(6) (2001) 627-634

17. Dai, F., Wu, J.: Distributed dominant pruning in ad hoc networks. In: Proceedings of the IEEE 2003 International Conference on Communications (ICC 2003). Volume 1. (2003) 353-357

18. Wu, J., Dai, F.: A generic distributed broadcast scheme in ad hoc wireless networks. IEEE Trans. Computers 53(10) (2004) 1343-1354

19. Lou, W., Wu, J.: On reducing broadcast redundancy in ad hoc wireless networks. IEEE Trans. on Mobile Computing 1(2) (2002) 111-123

20. Impett, M., Corson, M.S., Park, V.: A receiver-oriented approach to reliable broadcast in ad hoc networks. In: Proc. of Wireless Comm. and Networking Conf. (WCNC). Volume 1. (2000) 117-122

21. NS-2: The network simulator, http://www.isi.edu/nsnam/ns/ (2007)

22. de Waal, C.: A mobility scenario generation and analysis tool. www.informatik.unibonn.de/IV/BonnMotion/ (2007)

23. de Oliveira, T.B., Costa, V.F., Greve, F., Schnitman, L.: Evaluating the impact of faults on broadcasting protocols for manets. In: VII Workshop on Fault-Tolerant Computing, with (SBRC) Symp. on Computer Networks, Curitiba, Brazil (2006) 49-60

24. Wu, J., Dai, F.: Efficient broadcasting with guaranteed coverage in mobile ad hoc networks. IEEE Transactions on Mobile Computing 4(2) (2005) 259-270 
25. Pagani, E., Rossi, G.P.: Providing reliable and fault tolerant broadcast delivery in mobile ad-hoc networks. Mob. Netw. Appl. 4(3) (1999) 175-192

26. Kermarrec, A.M., Massoulié, L., Ganesh, A.J.: Probabilistic reliable dissemination in large-scale systems. IEEE Trans. Parallel Distrib. Syst. 14(3) (2003) 248-258

27. Lim, G., Shin, K., Lee, S., Yoon, H., Ma, J.S.: Link stability and route lifetime in ad-hoc wireless networks. In: Proc. of the 2002 Int. Conf. on Parallel Processing Workshops (ICPPW '02), Washington, DC, USA, IEEE Computer Society (2002) 116 